\title{
A Plaque-Type Solitary Reticulohistiocytoma in a Two-Year-Old Boy
}

\author{
Rintaro Shibuya $^{a}$ Hideaki Tanizaki ${ }^{a, b}$ Yo Kaku ${ }^{b}$ Masao Yonezawa ${ }^{b}$ \\ Yuri Ryu $^{a, b}$ Atsushi Otsuka $^{a}$ Kenji Kabashima ${ }^{a}$ Yoshiki Miyachi $^{a}$ \\ ${ }^{a}$ Department of Dermatology, Kyoto University Graduate School of Medicine, Kyoto, and \\ ${ }^{b}$ Department of Dermatology, Nagahama Red Cross Hospital, Nagahama, Japan
}

\section{Key Words}

Reticulohistiocytoma $\cdot$ Solitary plaque $\cdot$ Juvenile xanthogranuloma

\begin{abstract}
Reticulohistiocytoma $(\mathrm{RH})$ is a dermal histiocytic infiltration composed of large histiocytes with eosinophilic glassy cytoplasm. RH is classified into three clinical forms: solitary RH, diffuse cutaneous RH without systemic involvement and multicentric reticulohistiocytosis with systemic diseases. Solitary RH generally manifests as a nodular lesion in adults without accompanying systemic diseases. Herein, we describe a case of solitary RH with an atypical clinical manifestation as a red-brown-colored plaque in a 2-year-old boy. Atypical presentations of RH may pose diagnostic difficulty unless $\mathrm{RH}$ is considered. A correct diagnosis of RH can ensure avoidance of unnecessary invasive procedures.

(c) 2015 S. Karger AG, Basel
\end{abstract}

\section{Introduction}

Reticulohistiocytoma (RH) is a dermal histiocytic infiltration composed of large histiocytes with eosinophilic glassy cytoplasm. RH is classified into three clinical forms: solitary $\mathrm{RH}$, diffuse cutaneous RH without systemic involvement and multicentric reticulohistiocytosis with systemic diseases. Solitary RH usually presents as a small painless nodular lesion that can affect any part of the body, but with a predilection for the head and neck. Diffuse cutaneous RH presents as multiple nodular lesions which are usually located on the head and neck. Solitary and diffuse cutaneous RH do not accompany systemic diseases. In contrast, multicentric reticulohistiocytosis commonly manifests as multiple cutaneous histiocytic lesions on the face and acral sites complicated with systemic diseases and/or arthritis [1]. 
The pathophysiology of RH is yet to be clarified, but it is considered to be non-neoplastic and arising from macrophages rather than dendritic histiocytic cells [2]. Solitary RH generally manifests as a nodular lesion in adults [3]. Herein, we describe a case of solitary RH with an atypical clinical manifestation as a red-brown-colored plaque in a 2-year-old boy.

\section{Case Presentation}

A 2-year-old boy presented with a 4-month history of a solitary plaque on the abdomen without any symptoms. He had no relevant medical history and did not take any medications. There were no other remarkable clinical findings. The skin lesion exhibited a welldemarcated brown-red plaque measuring $15 \times 10 \mathrm{~mm}$ (fig. 1a). No family members exhibited similar symptoms.

Skin biopsy revealed nodular infiltration of large cells with an abundant eosinophilic ground-glass cytoplasm between collagen bundles in the superficial, partially vaguely nodular appearance (fig. 1b). Nuclei were generally vesicular with a single nucleolus and without atypia, but some were binucleated or multinucleated (fig. 1c). Lymphocytic infiltration was also noted. Histological differential diagnoses included Langerhans cell histiocytosis and non-Langerhans cell histiocytosis, such as RH or cutaneous Rosai-Dorfman disease. Immunohistochemical staining of the large cells was positive for CD68 (fig. 1d), but negative for S-100 protein and CD1a (data not shown). Langerhans cell histiocytosis is characterized by large cells with reniform nucleus and by both S-100 and CD1a positivity. Lack of emperipolesis and S-100 negativity excluded cutaneous Rosai-Dorfman disease. On the basis of the immunohistochemical findings along with large cells with an abundant eosinophilic groundglass cytoplasm, a diagnosis of solitary RH was made. No expansion or spontaneous regression of the lesion was observed over a 10-month follow-up even without surgical resection.

\section{Discussion}

RH was first described by Zak in 1950 [4]. Lesions are characterized by a dermal nodule consisting of histiocytic infiltration composed of large histiocytes with eosinophilic glassy cytoplasm. In one study of 44 cases of RH patient ranging from 2.5 to 74 years, 32 patients were 20-59 years old (mean 35 years) [3]. Although the etiology of RH remains unclear, it has been suggested that RH represents a non-neoplastic reactive process in which histiocytes respond to an unknown inflammatory stimulus [3]. With the exception of multicentric reticulohistiocytosis, RH usually involves skin and occasionally extends into the subcutis without accompanying systemic disease and/or arthritis. Spontaneous resolution without recurrence is the general outcome over a period of months to years and affected individuals remain healthy, while surgical resection is indicated for cosmetically disfiguring or symptomatic lesions [5]. Histiocytes of RH are usually positive for CD68, CD163 and alpha-1 antitrypsin, with erratic and focal staining for S-100 protein and microphthalmia-associated transcription factor [3]. They are negative for cytokeratin, CD1a, melan-A and HMB-45 and have a low Ki-67 index (<1\%); mitotic figures are occasionally observed [1, 3].

In addition to the early onset (at 2 years), the clinical manifestation of RH in our case was unique $[3,6]$. Solitary RH generally manifests as a nodule or a small superficial papule. Atypical presentations of RH may pose diagnostic difficulty unless RH is considered. A correct diagnosis of RH can ensure avoidance of unnecessary invasive procedures. 


\section{Case Reports in Dermatology}

\begin{tabular}{l|l}
\hline Case Rep Dermatol 2015;7:7-9 \\
\hline DOI: $10.1159 / 000371733$ & $\begin{array}{l}\text { C 2015 S. Karger AG, Basel } \\
\text { www.karger.com/cde }\end{array}$ \\
\hline
\end{tabular}

Shibuya et al.: A Plaque-Type Solitary Reticulohistiocytoma in a Two-Year-Old Boy

\section{References}

1 Zelger B, Cerio R, Soyer HP, Misch K, Orchard G, Wilson-Jones E: Reticulohistiocytoma and multicentric reticulohistiocytosis. Histopathologic and immunophenotypic distinct entities. Am J Dermatopathol 1994;16:577-584.

-2 Purvis WE 3rd, Helwig EB: Reticulohistiocytic granuloma (reticulohistiocytoma) of the skin. Am J Clin Pathol 1954;24:1005-1015.

-3 Miettinen M, Fetsch JF: Reticulohistiocytoma (solitary epithelioid histiocytoma): a clinicopathologic and immunohistochemical study of 44 cases. Am J Surg Pathol 2006;30:521-528.

4 Zak FG: Reticulohistiocytoma ('ganglioneuroma') of the skin. Br J Dermatol Syph 1950;62:351-355.

5 Toporcer MB, Kantor GR, Benedetto AV: Multiple cutaneous reticulohistiocytomas (reticulohistiocytic granulomas). J Am Acad Dermatol 1991;25:948-951.

6 Caputo R, Grimalt R: Solitary reticulohistiocytosis (reticulohistiocytoma) of the skin in children: report of two cases. Arch Dermatol 1992;128:698-699.
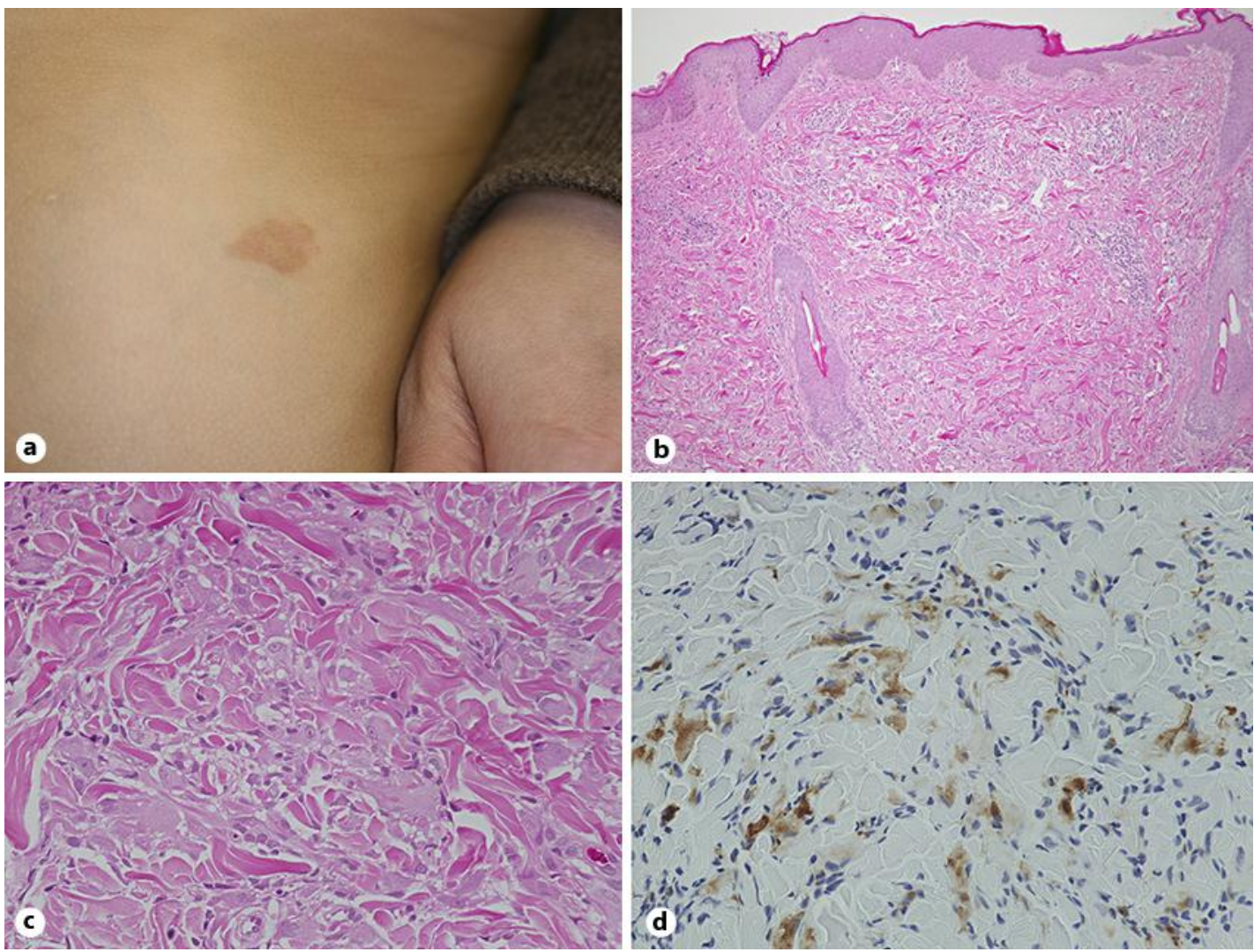

Fig. 1. Clinical appearance and histological findings. a Solitary, red-brown, well-demarcated plaque on the abdomen. b, c Nodular infiltration of large cells with eosinophilic ground-glass cytoplasm accompanied by scattered lymphocytes in the superficial and middle dermis (b: lower magnification, c: higher magnification). $\mathbf{d}$ The large epithelioid histiocytes in the dermis were positive for CD68. 